\title{
Bioorthogonal Modification of the Major Sheath Protein of Bacteriophage M13: Extending the Versatility of Bionanomaterial Scaffolds
}

\author{
Taylor Urquhart, Elisabeth Daub and John Frank Honek \\ Department of Chemistry, Waterloo Institute for Nanotechnology, University of Waterloo, 200 University Avenue, \\ Waterloo, Ontario, Canada N2L $3 \mathrm{G}_{1}$
}

Supporting Information Placeholder

\begin{abstract}
With a mass of $\sim 1.6 \times 10^{7}$ Daltons and composed of approximately 2700 proteins, bacteriophage M13 has been employed as a molecular scaffold in bionanomaterials fabrication. In order to extend the versatility of $\mathrm{M}_{13}$ in this area, residue-specific unnatural amino acid incorporation was employed to successfully display azide functionalities on specific solvent-exposed positions of the pVIII major sheath protein of this bacteriophage. Employing a combination of engineered mutants of the gene coding for the pVIII protein, the methionine (Met) analog, L-azidohomoalanine (Aha), and a suitable Escherichia coli Met auxotroph for phage production, conditions were developed to produce M13 bacteriophage labeled with over 350 active azides (estimated by fluorescent dye labeling utilizing a strain-promoted azidealkyne cycloaddition) and capable of azide-selective attachment to $5 \mathrm{~nm}$ gold nanoparticles as visualized by transmission electron microscopy. The capability of this system to undergo dual labeling utilizing both chemical acylation and bioorthogonal cycloaddition reactions was also verified. The above stratagem should prove particularly advantageous in the preparation of assemblies of larger and more complex molecular architectures based on the M13 building block.
\end{abstract}

Viral scaffolds are receiving intense interest for their use in materials and bionanomaterials ${ }^{1-16}$. Owing to its well-defined architecture and the ability to finely control its structure through molecular biological $^{17-19}$ as well as chemical methods $^{20-23}$, bacteriophage M13 has been employed in the fabrication of bionano-wires ${ }^{10}$, functional biomaterials ${ }^{4-9}$, sensors or probes ${ }^{1,12,15}$, and bioelectronic devices such as solar cells and piezoelectric biobatteries ${ }^{3,5,7,13}$. This filamentous virus (dimensions of approximately $6 \mathrm{~nm} \times 900 \mathrm{~nm}$ ), specific for Escherichia coli, is self-assembled at the bacterial membrane ${ }^{19,24}$. The mature bacteriophage is composed of five copies of each of four different tip proteins: pIII, pVI, pVII, pIX, and approximately 2700 copies of the major coat protein, pVIII, which is helically arranged around the encapsulated viral DNA genome (Figure 1) $)^{21,25}$. Modification of $\mathrm{M}_{13}$ with unnatural amino acids (UAA) has been accomplished on the pIII coat protein for phage display applications, although these studies did not assess the extent of UAA incorporation into M13 itself ${ }^{7,19,26,27}$. Given the relatively low copy number of pIII subunits per M13 phage, more highly func- tionalized nano-scale materials could result from the engineering of the major coat protein pVIII without perturbing the pIII protein, a common site for the display of peptides having affinities to xenomaterials. For (bio)materials applications of M13, knowledge of the extent of UAA incorporation into $\mathrm{M}_{13}$ and the development of a system for multiple labeling capabilities are also essential.

To achieve this, we show that the residue specific incorporation of $L$-azidohomoalanine (Aha), an analog of the amino acid methionine (Met), into Met positions in pVIII is possible when infecting a Met auxotrophic strain of E. coli (ER2738 $m e t E$ ) with engineered variants of M13 under specific media conditions. E. coli strain ER2738 was utilized to provide the F' required for $\mathrm{M}_{13}$ infection. As well, inactivation of the metE gene $\left(\Delta\right.$ metE774::kan) in ER2738 was constructed by $\mathrm{P}_{1 \mathrm{vir}}$ transduction to block $E$. coli Met biosynthesis and eliminate competition from endogenously biosynthesized methionine. Successful labeling of pVIII utilizing the displayed azide was accomplished using strain-promoted azide-alkyne cycloaddition (SPAAC; Figure 1$)^{28}$. The pVIII protein is highly tolerant to mutagenesis and contains only a few epitopes that result in a large loss of viability when changed ${ }^{29}$. Constructing several variants of $\mathrm{M}_{13}$ that repositioned a Met residue (and therefore the Aha analog) for optimum solvent exposure enhanced the availability of this azide group for phage surface modification on the mature pVIII coat protein (Figure 1).

Of the three Met residues present in pro-pVIII, two are removed by the host $E$. coli leader peptidase before phage extrusion (Figure 1). The Met residues at positions -5 and 28 were replaced by leucines prior to substituting Met at posi-

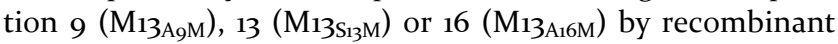
DNA techniques in order to counter any possible effects in phage production levels caused by substitutions by Aha at these non-strategic Met positions in pVIII. Net phage production for each variant was compared with that of WT M13 (M13wT) at 4.5 hours in order to obtain a measure of phage viability. The M13 variants produced under these conditions resulted in some loss of viability ranging from 3.0-fold to $7 \cdot 7^{-}$ fold compared to M13wt (Supplementary Figure 1a). Of the three single Met variants, M13 $\mathrm{A}_{16 \mathrm{M}}$ viability was the lowest. The A16M mutation might interfere with the stacking of adjacent pVIII subunits compared to positions $\mathrm{A}_{9}$ and $\mathrm{S}_{13}$ that are more exposed in the N-terminal region of pVIII. In a randomized mutagenesis study on pVIII, the positions $\mathrm{A}_{9}, \mathrm{~S}_{13}$ 
and A16 were found to be highly variable ${ }^{30}$. The mutability of pVIII was consistent with the good yields of variants produced in this current study.

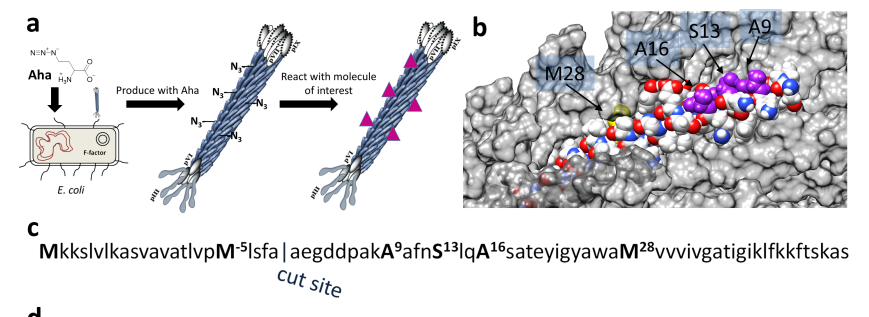

d

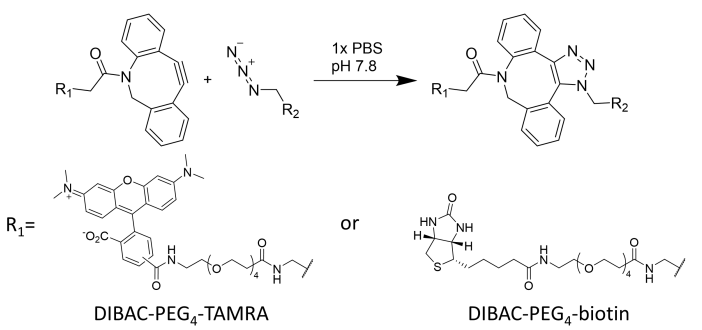

Figure 1: (a) Scheme showing the production of M13 phages with Aha and the modification of azide-containing Miz. (b) Molecular structure (PDB: $2 \mathrm{MJZ}$ ) of pVIII coat protein assembly showing the position of $\mathrm{A}_{9}, \mathrm{~S}_{13}$ and $\mathrm{A}_{16}$ residues in purple and $\mathrm{M}_{2} 8$ in yellow ${ }^{25}$. (c) Amino acid sequence of pVIII pro-coat protein with residues mutated in this study highlighted in bold. The leader peptidase cleavage site is also shown. (d) General scheme for the SPAAC reaction and bioorthogonal labeling agents utilized in this work.

With the availability of several viable bacteriophage variants containing relocated Met positions on their surface exposed pVIII proteins, investigations on unnatural amino acid bioincorporation into pVIII were undertaken. It has been established that Aha can compete with Met and be charged onto tRNA by methionyl-tRNA synthetase ${ }^{31}$. Notably, when an amino acid auxotrophic strain of $E$. coli is grown in media containing the UAA of interest, incorporation of the UAA occurs ${ }^{31-35}$. Additionally, recent metabolic engineering work has demonstrated that Aha can be intracellularly produced ${ }^{36}$. The added challenge of incorporation of Aha into M13 during the process of phage infection and replication, which includes the complex production of eleven different phage proteins (pI-pXI), some of which are not components of the mature M13 phage but are required to be functional, was successfully met by optimization of both Aha and Met levels in the bacterial growth medium (Supplementary Figure 1).

Incorporation of Aha into pVIII was confirmed by electrospray ionization mass spectrometry (ESI-MS). The major mass peak (5279 Da) observed for pVIII in a spectrum of M13AgM grown in the absence of Aha was consistent with the theoretical mass of $\mathrm{pVIII}_{\mathrm{AgM}}$ (528o Da; Figure 2 and Supplementary Figure 2). When M13A9M was produced in the presence of $10 \mathrm{mM}$ Aha and $235 \mu \mathrm{M}$ Met, an additional protein with a mass of 5274 Da was observed which corresponded to the expected mass of $\mathrm{PVIII}_{\mathrm{Ag} \mathrm{M}}$ containing Aha (Figure 2). This expected mass difference of ${ }_{-5}$ Da was also observed in the mass spectra of $\mathrm{pVIII}_{\mathrm{S} 13 \mathrm{M}}$ and $\mathrm{pVIII}_{\mathrm{WT}}$ analyzed from phage that were grown in the presence of Aha (Supplementary Figures 3 and 4 ). The relative intensities of the Aha-pVIII peaks were $10-13 \%$ of the Met-pVIII peak in each case. The negative control, M13M-5/28L, lacked the associated peak at -5
Da from the major pVIII peak (Supplementary Figure 5). A difference in mass of $5 \mathrm{Da}$ is only consistent with Aha substitution. As there is only a single Met in $\mathrm{pVIII}_{\mathrm{WT}}, \mathrm{pVIII}_{\mathrm{AgM}}$ and $\mathrm{pVIII}_{\mathrm{S}_{13} \mathrm{M}}$, Aha is expected to have occurred at positions 28, 9 and 13 on pVIII respectively.

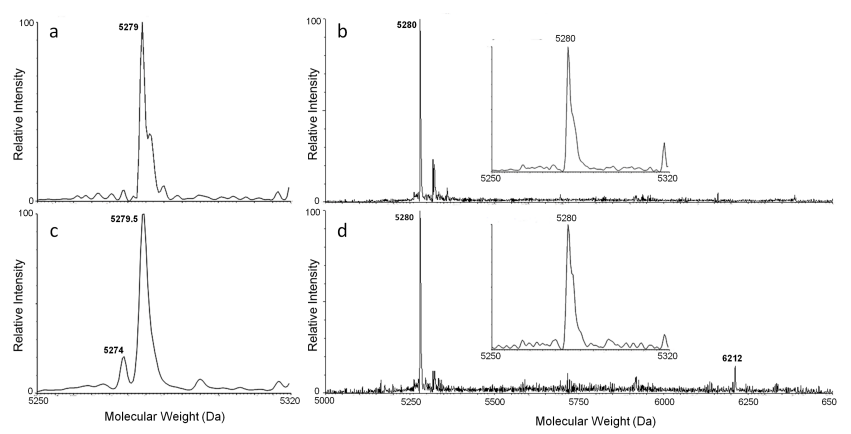

Figure 2: Deconvoluted mass spectra for M13AgM grown in the absence or presence of Aha from ESI-TOF MS experiments. As pVIII is the major M13 protein, mass spectral analyses readily detect this major component (a) M13AgM grown without Aha as outlined in the Methods. (b) M13AgM grown without Aha and then reacted with $30 \mu \mathrm{M}$ DIBAC-PEG $4^{-}$ TAMRA. The inset shows the portion of the spectrum from 5250 Da to 5320 Da. (c) M13AgM grown with $10 \mathrm{mM}$ Aha as outlined in the methods. (d) M13AgM grown with $10 \mathrm{mM}$ Aha and then reacted with $30 \mu \mathrm{M}$ DIBAC-PEG ${ }_{4}$ TAMRA. The inset shows the portion of the spectrum from 5250 Da to 5320 Da.

Critical to future applications of M13 as a multivalent nanoparticle is the proof-of-concept of successfully applying bioorthogonal-labeling chemistries to the above Ahacontaining bacteriophage ${ }^{31,37}$. The SPAAC reaction was utilized here with an aza-dibenzocycloocytne (DIBAC) reactive group, which was designed by Debets, M.F. and co-authors to have both fast kinetics and favourable solubility in water ${ }^{28}$. Aha-containing $\mathrm{M}_{13}$ was reacted with DIBAC-PEG $4_{-}^{-}$ tetramethylrhodamine (DIBAC-PEG ${ }_{4}$-TAMRA) and the absorbance spectra of the labeled phages were used to estimate the incorporation efficiency of Aha. Labeling of Mi3AgM was confirmed by mass spectrometry experiments (Figure 2 ). The very low TAMRA absorbance in phages grown in the absence of Aha indicated that the washing procedure effectively removed non-specifically bound DIBAC-PEG 4 -TAMRA (Figure 3). The TAMRA-labeled M13 phages exhibited a visible absorbance spectrum with a maximum at $556 \mathrm{~nm}$ and a shallow secondary maximum at $518 \mathrm{~nm}$, corresponding to the absorbance of TAMRA ${ }^{38,39}$. M13A9M and M13sisM were labeled equally well at $14 \pm 2 \%$ and $13 \pm 2 \%$ respectively and suggest that $13-14 \%$ of pVIII subunits had Aha incorporated. M13wT labeling was lower at $5 \pm 1 \%$ of pVIII subunits labeled. M13wT was not expected to react to completion since structural studies on pVIII have indicated that Met28 which is present in M13wT is not likely exposed to the solvent ${ }^{40}$. Labeling of M13wT in this study could be attributed to the flexibility of M13 itself allowing exposure of a fraction of the Aha28 residues for reaction ${ }^{41}$. The percentages of incorporation of Aha estimated here were similar to that observed for adenovirus coat protein labeling using a media replacement strategy, although in the current M13 study an intact bacteriophage has been labeled ${ }^{42}$. As a negative control, M13M-5/28L grown in $10 \mathrm{mM}$ Aha and $235 \mu \mathrm{M}$ Met was reacted with the same dye 
(Figure 3). Using the same mathematical treatment, the extent of labeling was estimated at $1.4 \pm 1 \%$. This control suggests that labeling with DIBAC is specific to pVIII with an azide incorporated. Furthermore, it was expected that this labeling strategy would be orthogonal to other common protein labeling strategies. Indeed, when carried out together with acylation by NHS-sulfo-cy7 (an amine specific reagent), dual-labeling of $\mathrm{M}_{13} \mathrm{AgM}^{-}$-Aha with both dyes occurred as expected. For A9M, dual-labeling was observed as evidenced by the TAMRA peak at $556 \mathrm{~nm}$ and the sulfo-cy7 peak at $750 \mathrm{~nm}$ (Figure 3e). Consistent with the other results for SPAAC labeling of $\mathrm{M}_{13}$ the labeling efficiency of DIBAC-TAMRA was $15.5 \%$ for dual-labeled M13. The M13M-5/28L negative control did not react with DIBAC-TAMRA at all regardless of the growth conditions (Figure 3f). As expected, NHS-sulfo-cy7 reacted with both variants and regardless of whether they were grown in the presence or absence of Aha. Dual-labeling on single M13 particles was also verified by dual-labeling followed by affinity isolation experiments. When DIBAC-biotin was reacted alongside NHS-sulfo-cy7 to produce dual-labeled A9M-Aha, the sulfo-cy7 absorbance signal could be used to track separation of biotinylated Miz using streptavidin (SA) magnetic particles (Supplemental Figures 14 and 15). These additional experiments are consistent with the occurrence of dual-labeling resulting in a population of M13 conjugated with two different labels on the same Miz particle.
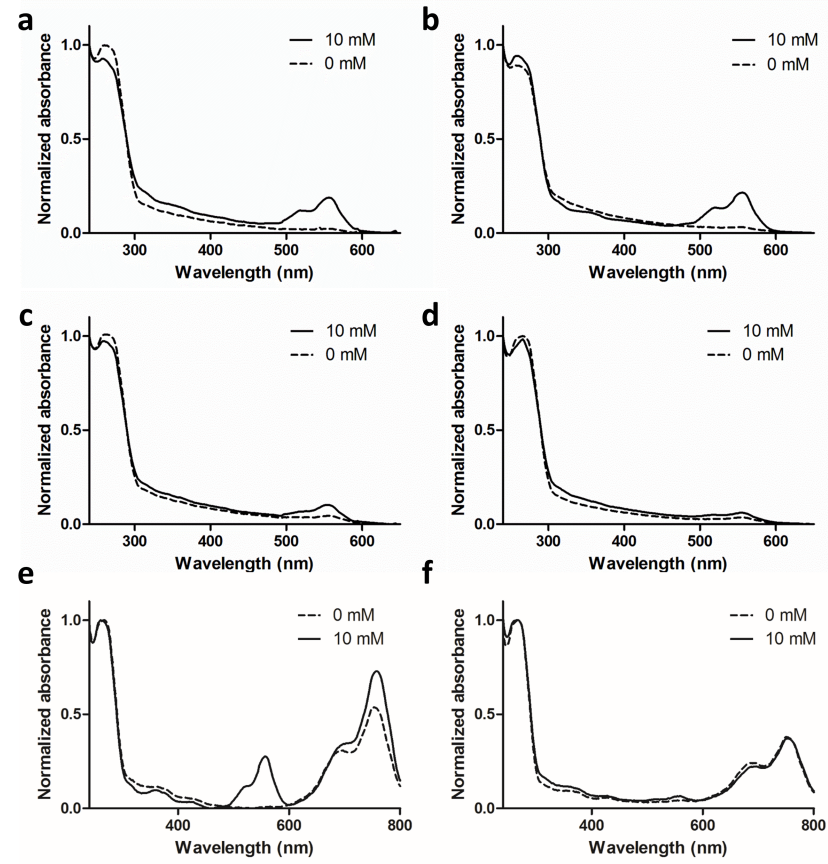

Figure 3: Absorbance spectra of M13 phages grown with 235 $\mu \mathrm{M}$ Met in the presence (10 $\mathrm{mM}$ ) or absence (o $\mathrm{mM}$ ) of Aha after labeling with DIBAC-PEG ${ }_{4}$-TAMRA. (a) M13AgM, (b) $\mathrm{M}_{13 \mathrm{~S}_{13} \mathrm{M}}$, (c) M13wT, or (d) M13M-5/28L were reacted for 24 hours

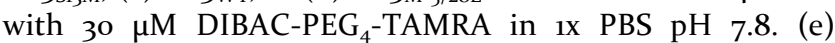
M13A9M or (f) M13M-5/28L were reacted for 24 hours with $30 \mu \mathrm{M}$

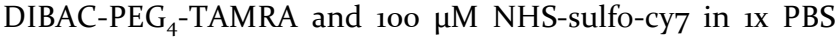
$\mathrm{pH}$ 7.8. Excess dye was removed using a $10 \mathrm{kDa}$ spin filtration column.

For visual inspection of bioorthogonal labeling using TEM, M13 variants with displayed azides were reacted with DIBAC-PEG 4 -biotin and incubated with streptavidin-labeled
AuNPs (SA-AuNPs). Uniformity of distribution of AuNPs was seen in M13A9M and M13si3M grown with $10 \mathrm{mM}$ Aha and 235 $\mu \mathrm{M}$ Met (Supplementary Figure 6 and Figure 4). When biotin-labeled $\mathrm{M}_{13}$ and SA-AuNPs were incubated together the average numbers of AuNPs observed bound to each phage particle were $54 \pm 19$ and $57 \pm 15$ for M13AgM and M13 $\mathrm{S}_{13 \mathrm{M}}$ respectively (Supplementary Figure 7). The differences in contrast seen in some images can be attributed to differences in stain density between grid preparations. In the samples grown in the absence of Aha, a small number of AuNPs can be seen associating with M13 phages, but substantially fewer than the Aha-labeled phages (Figure 4 and Supplementary Figure 6). In M13wt, as the azide would be positioned at the interface between pVIII subunits, it is possible that adduct formation interferes with the stability of assembled M13. In this regard the structures of M13wT phages post-reaction appeared twisted and aggregated (Supplementary Figure 8). As well, the extent of M13wt labeling was much lower than for the M13A9M and M13si3M variants (Supplementary Figure 7). The M13M-5/28L bacteriophage produced with Aha was only able to bind $6 \pm 3$ AuNPs per phage on average, likely due to non-specific interactions (Supplementary Figure 9). It is possible that the number of associated AuNPs visualized by TEM under-represents the actual number of pVIII subunits with incorporated Aha. Due to the relatively large size of the AuNPs, it is expected that not all Aha-pVIII subunits have a AuNP associated with them. The TEM results were also reflected in the mass spectra of biotin-labeled phages, in which only for M13A9M and M13 ${ }_{13} \mathrm{M}$ could the biotin label be detected (Supplementary Figures 10-13).

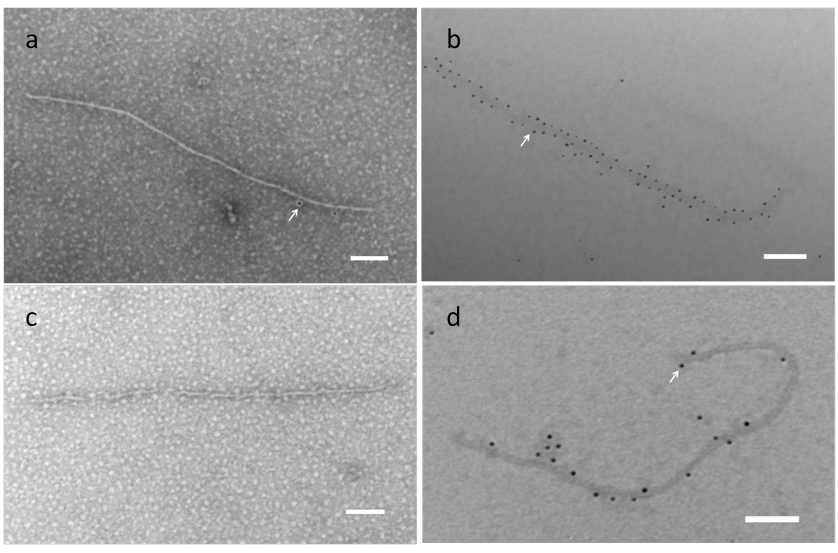

Figure 4: Transmission electron microscopy (TEM) images of M13si3M and M13wT constructs visualized with $5 \mathrm{~nm} \mathrm{SA-}$ AuNPs. Non-specific binding was blocked with the addition of $0.1 \%$ BSA. (a) M13 $\mathrm{S}_{13} \mathrm{M}$ grown in the absence of Aha and

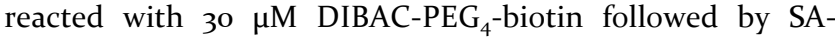
AuNP. (b) M13si3M grown with $10 \mathrm{mM}$ Aha/235 $\mu \mathrm{M}$ Met and reacted with $30 \mu \mathrm{M}$ DIBAC-PEG 4 -biotin followed by SAAuNP. (c) Mi3wt grown in the absence of Aha and reacted

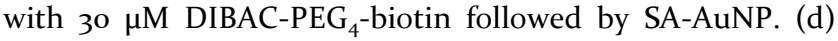
M13wT grown with $10 \mathrm{mM}$ Aha/235 $\mu \mathrm{M}$ Met and reacted with

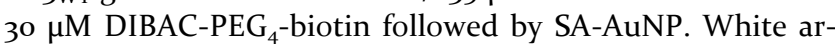
rows show AuNPs and the white scale bar shows $100 \mathrm{~nm}$.

The goal of this research was to develop an enabling M13 bioincorporation strategy which would provide labeling sites capable of modification by methods orthogonal to the standard chemical labeling methods that have been previously utilized $^{21}$. The research reported herein has successfully 
demonstrated UAA incorporation into the pVIII protein of an entire bacteriophage $\mathrm{M}_{13}$ and has demonstrated the capabilities of these phages to be further modified, providing enhanced versatility in a viral scaffold which frequently has been employed as a component in diverse applications ${ }^{1-16}$. This approach complements the currently available M13 toolset and provides additional possibilities for bottom-up biomaterial fabrication. Using orthogonal methods to organize varied components onto such a scaffold in a controlled manner is expected to introduce new possibilities to the field of phage-based bionanomaterials.

\section{ASSOCIATED CONTENT}

\section{Supporting Information}

This material is available free of charge via the Internet at http://pubs.acs.org.

Experimental procedures, mass spectra, transmission electron micrographs, characterization (Pdf)

\section{AUTHOR INFORMATION}

\section{Corresponding Author}

*jhonek@uwaterloo.ca

\section{Notes}

The authors declare no competing financial interests.

\section{ACKNOWLEDGMENT}

The authors gratefully acknowledge the research support provided by the Natural Sciences and Engineering Research Council of Canada (NSERC) (J.F.H.), University of Waterloo (Ontario, Canada) (J.F.H., T.U.), and a NSERC Canadian Graduate Scholarship (CGS-M), Ontario Graduate Scholarship and a Waterloo Institute for Nanotechnology Nanofellowship (T.U.). The authors would like to thank Dr. Richard Smith for electrospray ionization MS analysis, the Department of Biology for access to the TEM facility and Ms. Natalie Koay and Dr. Zhengding Su for early studies on $\mathrm{M}_{13}$ in our laboratory.

\section{REFERENCES}

(1) Bardhan, N. M., Ghosh, D., and Belcher, A. M. (2014) Carbon nanotubes as in vivo bacterial probes. Nat. Commun. 5, 4918.

(2) Cao, B., Yang, M., and Mao, C. (2016) Phage as a Genetically Modifiable Supramacromolecule in Chemistry, Materials and Medicine. Acc. Chem. Res. 49, 1111-1120.

(3) Chen, P.-Y., Dang, X., Klug, M. T., Qi, J., Dorval Courchesne, N.-M., Burpo, F. J., Fang, N., Hammond, P. T., and Belcher, A. M. (2013) Versatile three-dimensional virusbased template for dye-sensitized solar cells with improved electron transport and light harvesting. ACS Nano 7, 65636574 .

(4) Chiang, C.-Y., Mello, C. M., Gu, J., Silva, E. C., Van Vliet, K. J., and Belcher, A. M. (2007) Weaving genetically engineered functionality into mechanically robust virus fibers. Adv. Mater. 19, 826-832.

(5) Jeong, C. K., Kim, I., Park, K.-I., Oh, M. H., Paik, H., Hwang, G.-T., No, K., Nam, Y. S., and Lee, K. J. (2013) Virusdirected design of a flexible $\mathrm{BaTiO}_{3}$ nanogenerator. ACS Nano 7, 11016-11025.
(6) Kim, W.-G., Kim, K., Ha, S.-H., Song, H., Yu, H.-W., Kim, C., Kim, J.-M., and Oh, J.-W. (2015) Virus based Full Colour Pixels using a Microheater. Sci. Rep. 5, 13757.

(7) Lee, B. Y., Zhang, J., Zueger, C., Chung, W.-J., Yoo, S. Y., Wang, E., Meyer, J., Ramesh, R., and Lee, S.-W. (2012) Virusbased piezoelectric energy generation. Nat. Nanotechnol. 7, $351-356$.

(8) Lee, J. Y., Chung, W.-J., and Kim, G. (2016) A mechanically improved virus-based hybrid scaffold for bone tissue regeneration. RSC Adv. 6, 55022-55032.

(9) Murugesan, M., Abbineni, G., Nimmo, S. L., Cao, B., and Mao, C. (2013) Virus-based photo-responsive nanowires formed by linking site-directed mutagenesis and chemical reaction. Sci. Rep. 3, 1820.

(10) Nam, K. T., Kim, D.-W., Yoo, P. J., Chiang, C.-Y., Meethong, N., Hammond, P. T., Chiang, Y.-M., and Belcher, A. M. (2006) Virus-enabled synthesis and assembly of nanowires for lithium ion battery electrodes. Science 312, $885-888$.

(11) Niu, Z., Bruckman, M. A., Harp, B., Mello, C. M., and Wang, Q. (2008) Bacteriophage M13 as a scaffold for preparing conductive polymeric composite fibers. Nano Res. $1,235-241$.

(12) Oh, J.-W., Chung, W.-J., Heo, K., Jin, H.-E., Lee, B. Y., Wang, E., Zueger, C., Wong, W., Meyer, J., Kim, C., et al. (2014) Biomimetic virus-based colourimetric sensors. Nat. Commun. 5, 3043

(13) Shin, D.-M., Han, H. J., Kim, W.-G., Kim, E., Kim, C., Hong, S. W., Kim, H. K., Oh, J.-W., and Hwang, Y.-H. (2015) Bioinspired piezoelectric nanogenerators based on vertically aligned phage nanopillars. Energy Environ. Sci. 8, 3198-3203.

(14) Wang, J., Yang, M., Zhu, Y., Wang, L., Tomsia, A. P., and Mao, C. (2014) Phage nanofibers induce vascularized osteogenesis in ${ }_{3} \mathrm{D}$ printed bone scaffolds. Adv. Mater. 26, 4961-4966.

(15) Wang, Y., Ju, Z., Cao, B., Gao, X., Zhu, Y., Qiu, P., Xu, H., Pan, P., Bao, H., Wang, L., et al. (2015) Ultrasensitive rapid detection of human serum antibody biomarkers by biomarker-capturing viral nanofibers. ACS Nano 9, 44754483.

(16) Zhu, H., Cao, B., Zhen, Z., Laxmi, A. A., Li, D., Liu, S., and Mao, C. (2011) Controlled growth and differentiation of MSCs on grooved films assembled from monodisperse biological nanofibers with genetically tunable surface chemistries. Biomaterials 32, 4744-4752.

(17) Lim, R. K., Li, N., Ramil, C. P., and Lin, Q. (2014) Fast and Sequence-Specific Palladium-Mediated Cross-Coupling Reaction Identified from Phage Display. ACS Chem. Biol. 9, 2139-2148.

(18) Seker, U. O. S., and Demir, H. V. (2011) Material binding peptides for nanotechnology. Molecules 16, 14261451.

(19) Tian, F., Tsao, M.-L., and Schultz, P. G. (2004) A phage display system with unnatural amino acids. J. Am. Chem. Soc. 126, 15962-15963.

(20) Kitov, P. I., Vinals, D. F., Ng, S., Tjhung, K. F., and Derda, R. (2014) Rapid, hydrolytically stable modification of aldehyde-terminated proteins and phage libraries. J. Am. Chem. Soc. 136, 8149-8152.

(21) Li, K., Chen, Y., Li, S., Nguyen, H. G., Niu, Z., You, S., Mello, C. M., Lu, X., and Wang, Q. (2010) Chemical modification of $\mathrm{M}_{13}$ bacteriophage and its application in cancer cell imaging. Bioconjugate Chem. 21, 1369-1377. 
(22) Mohan, K., and Weiss, G. A. (2016) Chemically Modifying Viruses for Diverse Applications. ACS Chem. Biol. 11, 1167-1179.

(23) Ng, S., Jafari, M. R., and Derda, R. (2011) Bacteriophages and viruses as a support for organic synthesis and combinatorial chemistry. ACS Chem. Biol. 7, 123-138.

(24) Williams, K. A., Glibowicka, M., Li, Z., Li, H., Khan, A. R., Chen, Y. M., Wang, J., Marvin, D. A., and Deber, C. M. (1995) Packing of Coat Protein Amphipathic and Transmembrane Helices in Filamentous Bacteriophage M13: Role of Small Residues in Protein Oligomerization. J. Mol. Biol. 252, 6-14.

(25) Morag, O., Sgourakis, N. G., Baker, D., and Goldbourt, A. (2015) The NMR-Rosetta capsid model of $\mathrm{M}_{13}$ bacteriophage reveals a quadrupled hydrophobic packing epitope. Proc. Natl. Acad. Sci. 112, 971-976.

(26) Liu, C. C., Mack, A. V., Tsao, M.-L., Mills, J. H., Lee, H. S., Choe, H., Farzan, M., Schultz, P. G., and Smider, V. V. (2008) Protein evolution with an expanded genetic code. Proc. Natl. Acad. Sci. 105, 17688-17693.

(27) Sandman, K. E., Benner, J. S., and Noren, C. J. (2000) Phage display of selenopeptides. J. Am. Chem. Soc. 122, 96o961.

(28) Debets, M. F., Berkel, S. S. van, Schoffelen, S., Rutjes, F. P., Hest, J. C. van, and Delft, F. L. van. (2010) Azadibenzocyclooctynes for fast and efficient enzyme PEGylation via copper-free $(3+2)$ cycloaddition. Chem. Commun. 46, 97-99.

(29) Roth, T. A., Weiss, G. A., Eigenbrot, C., and Sidhu, S. S. (2002) A minimized M13 coat protein defines the requirements for assembly into the bacteriophage particle. $J$. Mol. Biol. 322, 357-367.

(30) Held, H. A., and Sidhu, S. S. (2004) Comprehensive mutational analysis of the M13 major coat protein: improved scaffolds for C-terminal phage display. J. Mol. Biol. 340, 587597.

(31) Kiick, K. L., Saxon, E., Tirrell, D. A., and Bertozzi, C. R. (2002) Incorporation of azides into recombinant proteins for chemoselective modification by the Staudinger ligation. Proc. Natl. Acad. Sci. 99, 19-24.

(32) Duewel, H. S., Daub, E., Robinson, V., and Honek, J. F. (2001) Elucidation of solvent exposure, side-chain reactivity, and steric demands of the trifluoromethionine residue in a recombinant protein. Biochemistry 40, 13167-13176.
(33) Hest, J. C. van, Kiick, K. L., and Tirrell, D. A. (200o) Efficient incorporation of unsaturated methionine analogues into proteins in vivo. J. Am. Chem. Soc. 122, 1282-1288.

(34) Vaughan, M. D., Cleve, P., Robinson, V., Duewel, H. S., and Honek, J. F. (1999) Difluoromethionine as a novel $19 \mathrm{~F}$ NMR structural probe for internal amino acid packing in proteins. J. Am. Chem. Soc. 121, 8475-8478.

(35) Walasek, P., and Honek, J. F. (2005) Nonnatural amino acid incorporation into the methionine 214 position of the metzincin Pseudomonas aeruginosa alkaline protease. BMC Biochem. 6, 21.

(36) Ma, Y., Biava, H., Contestabile, R., Budisa, N., and Di Salvo, M. L. (2014) Coupling bioorthogonal chemistries with artificial metabolism: intracellular biosynthesis of azidohomoalanine and its incorporation into recombinant proteins. Molecules 19, 1004-1022.

(37) Patel, K. G., and Swartz, J. R. (2011) Surface functionalization of virus-like particles by direct conjugation using azide-alkyne click chemistry. Bioconjugate Chem. 22, 376-387.

(38) Meadows, D. L., Shafer, J. S., and Schultz, J. S. (1991) Determining the extent of labeling for tetramethylrhodamine protein conjugates. J. Immunol. Methods 143, 263-272.

(39) Valdes-Aguilera, O., and Neckers, D. C. (1989) Aggregation phenomena in xanthene dyes. Acc. Chem. Res. 22, 171-177.

(40) Marvin, D., Welsh, L., Symmons, M., Scott, W., and Straus, S. (2006) Molecular structure of fd (fi, Mis) filamentous bacteriophage refined with respect to X-ray fibre diffraction and solid-state NMR data supports specific models of phage assembly at the bacterial membrane. J. Mol. Biol. 355, 294-309.

(41) Beck, K., and Duenki, R. M. (1990) Flexibility of bacteriophage M13: Comparison of hydrodynamic measurements with electron microscopy. J. Struct. Biol. 105, 22-27.

(42) Banerjee, P. S., Ostapchuk, P., Hearing, P., and Carrico, I. S. (2011) Unnatural amino acid incorporation onto adenoviral (Ad) coat proteins facilitates chemoselective modification and retargeting of Ad type 5 vectors. J. Virol. 85 , 7546-7554. 
Authors are required to submit a graphic entry for the Table of Contents (TOC) that, in conjunction with the manuscript title, should give the reader a representative idea of one of the following: A key structure, reaction, equation, concept, or theorem, etc., that is discussed in the manuscript. Consult the journal's Instructions for Authors for TOC graphic specifications.

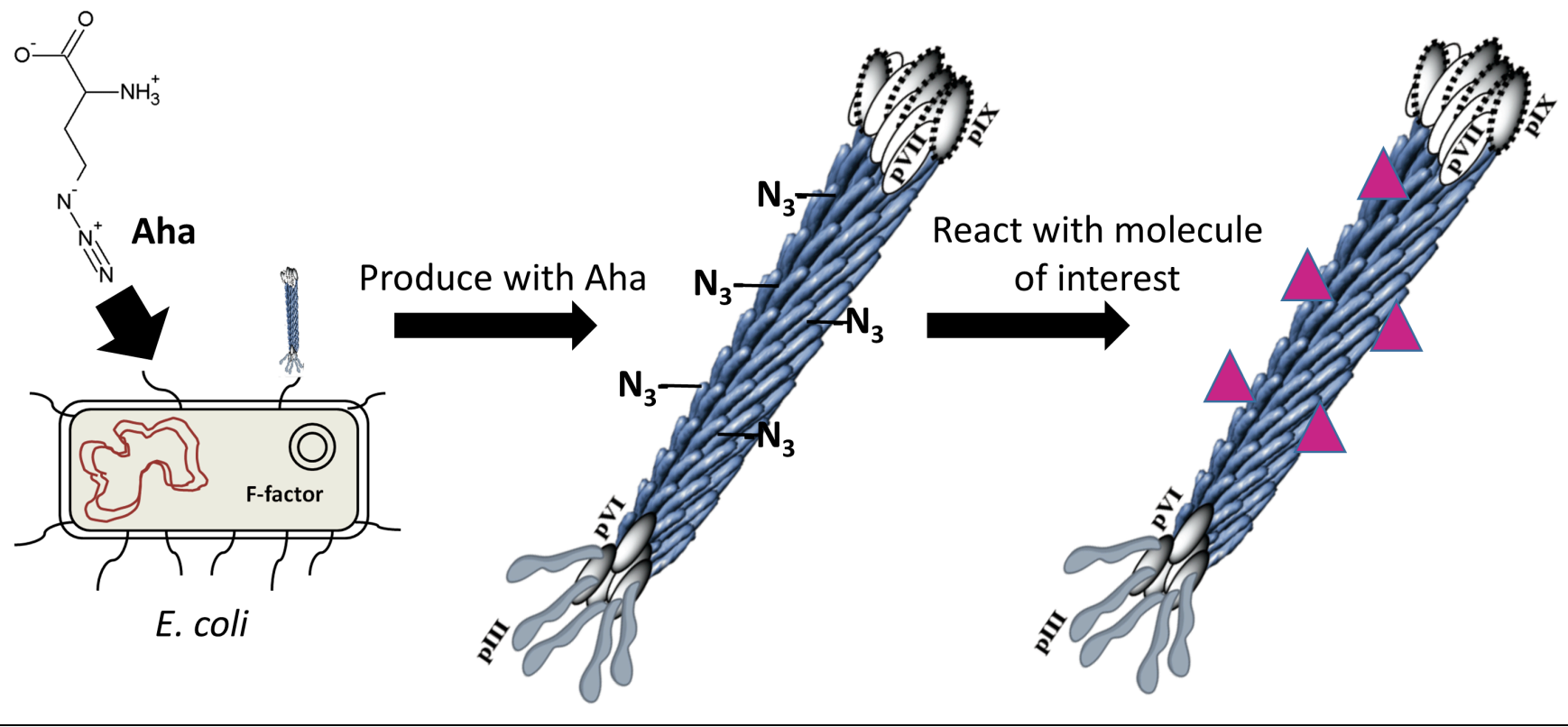

6 
This document is the unedited Author's version of a Submitted Work that was subsequently accepted for publication in Biconjugate Chemistry, copyright $\odot$ American Chemical Society after peer review. To access the final edited and published work see http://dx.doi.org/10.1021/acs.bioconjchem.6b00460. 